\title{
BMJ Global Health Changing stigmatisation of leprosy: an exploratory, qualitative life course study in Western Nigeria
}

\author{
Bassey Ebenso, ${ }^{\oplus 1}$ James Newell, ${ }^{2}$ Nick Emmel, ${ }^{3}$ Gbenga Adeyemi, ${ }^{4}$ Bola Ola ${ }^{5}$
}

To cite: Ebenso B, Newell J, Emmel N, et al. Changing stigmatisation of leprosy: an exploratory, qualitative life course study in Western Nigeria. BMJ Glob Health 2019;4:e001250. doi:10.1136/ bmjgh-2018-001250

Handling editor Stephanie M Topp

- Additional material is published online only. To view please visit the journal online (http://dx.doi.org/10.1136/ bmjgh-2018-001250).

Received 23 October 2018 Revised 29 December 2018 Accepted 10 February 2019

Check for updates

C Author(s) (or their employer(s)) 2019. Re-use permitted under CC BY-NC. No commercial re-use. See rights and permissions. Published by BMJ.

For numbered affiliations see end of article.

Correspondence to

Dr Bassey Ebenso;

b.e.ebenso@leeds.ac.uk

\section{ABSTRACT}

Introduction Renewed interest in health-related stigma has invigorated calls to understand factors and processes underlying stigma. However, few empirical studies explore the influences of structural discrimination and moral status on leprosy-related stigma. We investigated how sociocultural context and organisational policies and practices influenced the connotations of leprosy, sources of stigma and the changing social responses to leprosy in Western Nigeria. Methodology Ethnographic research conducted between 2008 and 2012 combined documents review with life history interviews of 21 individuals affected by leprosy and semistructured interviews with 26 community members in Western Nigeria. Interviews were audiotaped, transcribed verbatim and coded. Theoretical frameworks used to deepen social understandings of leprosy and responses to stigma included Link's and Phelan's conceptualisation of stigma and the concepts of structural discrimination and moral status.

Results Findings showed that connotations of leprosy in Yorùbá culture included the following: (i) perception of leprosy as the most shameful and detested condition and (ii) symbolic association with filth and immoral behaviour that is dishonouring to Yorùbá identity. Secondary analysis of archival materials revealed four sources of stigma: cultural beliefs about leprosy, health promotion messages embedded in primary school books, religious teachings about leprosy and campaigns conducted by the leprosy service in 1950s. Contrary to the portrayal of Yorùbá attitudes to leprosy as entirely negative, we identified that people affected by leprosy were creating new life courses to counter existing cultural accounts of marginalisation. Emerging narratives of inclusion outlined five facilitators of acceptance namely, antileprosy treatment, good moral character, supportive family networks, livelihoods, and contribution to community survival.

Conclusion Gaps highlighted by this study suggest that the global target of zero stigma and discrimination of leprosy will remain unattainable without better understanding of cultural significance(s) of leprosy and the local sources and underlying drivers of stigma that are crucial for developing context-specific stigma reduction interventions.

\section{INTRODUCTION}

Leprosy has been associated with stigmatisation throughout history despite being one of the least contagious of infectious diseases. ${ }^{1-3}$

\section{Key questions}

What is already known?

- There has been huge global success in reducing the burden of leprosy using multidrug therapy to treat leprosy.

- Progress towards stopping discrimination and promoting inclusion of people affected by leprosy has not kept pace with medical advances.

- There is an increasing interest in understanding the processes underlying stigmatisation of leprosy among programme implementers and researchers, though many stigma studies ignore the moral experiences of people affected by leprosy and the influence of sociocultural contexts on stigmatisation.

\section{What are the new findings?}

- This study provides rich contextualised understandings of Yorùbá ideas of leprosy, illuminating how culture and wider social factors such as social structures, policies and contact with Western missionaries shape people's diverse experiences and responses to leprosy-related stigma.

What do the new findings imply?

- The findings can contribute to better understanding of the sources and implications of leprosy-related stigma and to providing evidence for decision-making and practice among the Yorùbá people.

The disease is characterised by skin lesions and peripheral nerve damage that may lead to skin ulceration and physical deformities. Since the introduction of antibiotic multidrug therapy (MDT) for leprosy treatment in the 1980s, the number of new diagnosed cases has decreased, and leprosy was even declared eliminated as a public health problem at a global level in the year 2000, that is, $<1$ case per 10000 people. $^{4}$ Subsequently, global leprosy strategies have focused on reducing disease burden measured in terms of new cases with visible deformities. ${ }^{4}$ In keeping with this, the targets of the global leprosy strategy 2016-2020 'Accelerating towards a leprosy-free world' include the following: (i) zero visible disabilities among children 
and (ii) zero stigma and discrimination while ensuring that countries repeal discriminatory laws. ${ }^{5}$ However, scholars have contended that these targets are difficult to achieve in the near future and that leprosy will continue to impose huge burdens of disability and stigmatisation in many endemic countries ${ }^{67}$ with weak health systems. Consistent with these concerns, the global number of new diagnosed cases has remained above 200000 annually for the last decade, due, in part, to continuing stigmatisation and inadequate resources that hinder diagnosis and treatment of leprosy and perpetuate the spread of leprosy. ${ }^{5}$

Renewed emphasis on social aspects of leprosy has invigorated investigations into factors and processes underlying leprosy-related stigma. Some scholars have explored the causes and consequences of leprosy-related stigma ${ }^{89}$ or specific aspects such as concealment of leprosy. ${ }^{1011}$ Other scholars developed and validated tools to measure stigma or its proxies in different settings. ${ }^{12} 13$ However, many stigma studies in Nigeria have focused on surveying the knowledge, attitudes and practices (KAP) of health workers, students and the public in southeastern, northern and the middle belt of Nigeria, partly due to the ease of access to these groups of stakeholders. Overall, many of the studies showed mixed results. While some researchers blamed stigmatising attitudes on low levels of leprosy awareness of respondents, ${ }^{14-17}$ others argued that high levels of awareness among health workers and students were unmatched by positive attitudes. ${ }^{18} 19$ Although KAP surveys and using validated tools to measure stigma are important in their own right, Kleinman and Hall-Clifford argue that the first focus of the efforts to counteract stigma should be directed at exploring the underlying social and cultural processes of stigmatisation in the live worlds of the stigmatised. ${ }^{20}$ Kleinman and Hall-Clifford argue that ethnographic methods are better suited to unravelling the local value systems and what matters most in the live worlds of stigmatised groups, compared with using standard survey instruments.

Nigeria detects 3500 new leprosy cases annually, 25\% of whom suffer some impairment. ${ }^{21}$ There are significant geographical differences in the distribution of leprosy in Nigeria with notification rates higher in the North than in the South, and higher in the East than in the West of Nigeria. ${ }^{22}$ Those affected by leprosy continue to experience intense stigma and discrimination, ${ }^{23}$ particularly in Western Nigeria, home to the Yorùbá people. While we focus on Nigeria, the population of the Yorùbá in West Africa is estimated to be over 43 million, making them one of the largest groups in sub-Saharan Africa. Their main concentration is in southwest Nigeria where they number approximately 40 million (or $21 \%$ of Nigeria's population), occupying seven of Nigeria's 36 States: Ekiti, Lagos, Kwara, Ogun, Ondo, Osun and Oyo. ${ }^{24 p 35}$ However, despite being one of the largest groups in sub-Saharan Africa, little is known about the anthropology of leprosy among the Yorùbá people. This paper therefore explores the influence of cultural context and organisational policies and practices on the moral definition of leprosy and its stigma among the Yorùbá in Western Nigeria. The paper follows a reanalysis of transcripts of lived experiences of leprosy, conducted as part of an ethnographic study of the sources, severity and persistence of stigma in Western Nigeria, a part of which has been reported elsewhere. $^{25}$ That preliminary report (https://doi. org/10.1080/13696815.2012.704263) highlighted two key findings. First, it showed that the context in which people affected by leprosy found themselves in Western Nigeria was saturated in metaphorical language. That article documented how disease metaphors were used to codify and express Yorùbá understandings about leprosy. Second, it highlighted that information concerning Yorùbá understanding of leprosy (ie, about its causes, transmission, symptoms, treatment and cure) was lacking from the international literature. This paper fills the identified knowledge gap by answering three research questions: What are the moral connotations and social expectations regarding leprosy in Western Nigeria; what are the perceptions of symptoms and signs, causes and treatment of leprosy among the Yorùbá people and to what extent has stigmatisation of leprosy changed in Western Nigeria?

We begin by elaborating the methods used including the study design, data collection and theoretical models that informed data analysis. Our findings are presented according to six broad themes: (i) connotations to leprosy, (ii) causes and transmission of leprosy, (iii) the sources of stigma, (iv) Yorùbá perception of treatment and cure, (v) help-seeking for leprosy and (vi) the changing stigmatisation of leprosy. The views and experiences of people with leprosy and community members are elaborated, underlining those that are relevant for stigma reduction and achieving the global target of zero stigma and discrimination. We conclude by comparing our findings to social responses to leprosy in India, in Mali, West Africa and in Tanzania, East Africa.

\section{METHODS \\ Study design}

This study adopted a qualitative, retrospective narrative life course design to understand how leprosy shaped the lives of affected persons in Western Nigeria. Life course research is the study of social processes that extend over an individual's life span, or significant portions of it, especially relating to their family cycle, educational and training histories, as well as employment and occupational careers. ${ }^{26}$ Among other things, life course is shaped by cultural beliefs about the individual biography, institutionalised sequences of roles and positions, legal role restrictions and decisions of individual actors. A life course approach therefore aims to (i) provide better descriptions and explanations of life events and social patterns of life trajectories within a common conceptual and empirical frameworks; (ii) represent the processes 
that shape these events and trajectories and (iii) link these processes together and identify societal change or dynamics. For this study, life course experiences of living with leprosy incorporated the events and social patterns of the life trajectories of individuals affected by leprosy, beginning from the periods before leprosy diagnosis, extending through treatment for leprosy and discharge from treatment, to the moment they participated in the study.

\section{Study setting, target population and research team}

Our ethnographic study was implemented in Kwara and Oyo States that lie to the west and southwest, respectively, of Nigeria's capital, Abuja. Kwara and Oyo were selected because they had not participated in stigma studies prior to our research. A leprosy settlement in each state served as study sites, as most people with leprosy resided in settlements. A recent count suggests approximately 1600 people live in settlements in the two states with 768 (48\%) of them having visible impairment. Residents of leprosy settlements were mainly traders, farmers and hospital workers. The samples for the study were as follows: (i) individuals affected by leprosy who had completed a course of antileprosy treatment and resided in leprosy settlements in the study area and (ii) community members who did not have leprosy, purposively selected to provide information on the meanings and sociocultural perceptions of leprosy, and Yorùbá responses to leprosy. To capture the variations in community views, participants were purposively selected by age, gender, religion and their occupation or role in the community.

Two research assistants, who were community-based rehabilitation workers at the study sites, approached potential participants (face-to-face) to explain the study objectives and distribute participant information sheets (in local language), to help them to decide to participate (or not) in the study. To minimise the possibility of coercion, potential participants were then approached by a research assistant 2 days after explaining the research objectives and invited to participate in the study. Research assistants also obtained permission from heads of leprosy settlements to implement the study. The assistants received a 3-day introduction to social research and the life course approach, followed by a 2-day pilot study to check study feasibility and appropriateness of interview questions in Yorùbá culture. Interviews were conducted in Yorùbá language by $\mathrm{BE}$ and GA, who were male, postgraduate sociologists experienced in stigma research.

\section{Data collection}

Data collection consisted of document reviews and in-depth interviews with research participants. Three types of documents and texts provided a rich source of information for the study. First, historical materials from books and published articles downloaded from major databases of medical and social sciences. Second, leprosy control policies, records and reports on the organisation and implementation of leprosy control. Third, archival records from leprosy referral hospitals, the libraries of universities and voluntary agencies to illuminate the influence of institutional practices on leprosy-related stigma in Nigeria. ${ }^{27-29}$

Only one potential participant declined to participate in the study. Semistructured interviews were conducted in two phases of fieldwork, to unravel how people made sense of their experiences of and responses to leprosy and stigma across the life course in Western Nigeria. During phase 1 (September and October 2009), 36 semistructured interviews were conducted: 21 with people affected by leprosy in their homes, and 15 with community members aged $\geq 40$ years. Individuals affected by leprosy, interviewed for 30-45 min per person, were asked to reflect on their life histories and how living with leprosy and its stigma influenced their life trajectories including how leprosy affected family relationships, educational and training histories, as well as occupations. On the other hand, community members, interviewed for 15-20 min per person, were asked about the local notions of leprosy, and attitudes and responses to leprosy and affected persons. Phase 2 fieldwork (August 2010 to January 2011) served two main purposes. First, to conduct 11 additional semistructured interviews with community members aged $<40$ years (at their workplaces or schools) to investigate aspects of leprosy and stigma that emerged from analysis of phase 1 interviews. Second, using structured vignettes ${ }^{30}$ to crosscheck our interpretation of phase 1 interviews with research participants, that is, member checking. ${ }^{30}{ }^{31}$ In all, 12 of 36 participants interviewed in phase 1 were selected for member checking (seven affected individuals and five community members). The 12 participants were purposively selected to illuminate issues raised in the vignettes.

\section{Data analysis}

All interviews were audio-recorded, transcribed verbatim and coded for manual analysis. During coding, BE and GA organised segments or paragraphs of data into 'chunks' before assigning meanings to chunks. The chunks of material were then labelled by research question and categorised with a term based on the actual language of study participants. The research involved a process of concurrent interviewing, constant comparative analysis, interpretation and reflection on research questions and data collected, asking analytic questions, and writing reflexive memos throughout the study. A reanalysis of archival materials and interview transcripts that informed this article was undertaken between January and July 2017.

\section{Reflexivity}

$\mathrm{BE}, \mathrm{GA}$ and $\mathrm{BO}$ were born in Western Nigeria and had extensive engagement with the study region, both as researchers (BE and GA) and as global health practitioners (BE and $\mathrm{BO})$. These roles aided our understanding of the sociocultural context of Western Nigeria and enhanced awareness of, and sensitivity to the 
challenges, decisions and issues encountered in the study region. The roles also facilitated working with research participants and informants during the study. These previous experiences and insight from the literature meant that we brought certain biases to the study. Although every effort has been made to ensure objectivity, we admit that these biases have shaped our experiences of this study and the way that we viewed and understood the data collected and interpreted. This reflexivity guided the analysis and discussion of study finding in the rest of this paper thesis.

\section{Theoretical models of stigma that informed data analysis}

In framing our study of stigma, we drew on a number of theoretical models that help to account for social, cultural and material contexts of interaction. ${ }^{32-35}$ Goffman argued that stigma is embedded in relationships rather attributes, stating that stigma occurs when the construction of social categories is linked to stereotypical beliefs that label and distinguish people or groups for being different or unacceptable, thereby 'spoiling their social identity'. According to Goffman, stigmatised persons either internalised the prevailing negative beliefs about their identities and reputations or resisted stigma by using cover-up strategies such as stories to deflect attention from tainted identities. Extending Goffman's idea of relationships, contemporary stigma theories argue that stigmatisers exploit situations of unequal power within their sociocultural environment to control, regulate or exclude others for breaching social norms or expectations. ${ }^{34}$ Link and Phelan regard stigma as a social construction that occurs when the processes of labelling, negative stereotyping, exclusion, discrimination and low status coexist in a power situation that facilitates the manifestation of these processes. ${ }^{34}$ Using a dimensional conceptualisation of stigma, Jones et $a \vec{l}^{7}$ described six dimensions that influenced societal responses to stigma. The dimensions are as follows: (i) 'concealability'perception that the degree of visibility of a difference is linked to the severity of stigmatisation; (ii) 'peril'- the perception that the stigma is threatening to others or has the potential for contagion; (iii) 'chronicity'-conditions with longer duration/courses attract severer stigma compared with conditions that are of shorter duration; (iv) 'disruptiveness' - the extent to which a difference interferes with smooth social interaction; (v) aestheticsthe potential for a condition to induce a disgust reaction and (vi) 'origin'- the perception that a stigma is caused deliberately, unintentional or present at birth. ${ }^{38}$ Scholars have used Jones $e t a l$ s framework to examine how each of these six dimensions affected stigmatisers' perception of stigmatised individuals, with concealability and origin receiving the most empirical attention. ${ }^{39}$

To elucidate the processes of institutional stigma, Corrigan $e t a t^{40}$ adopted the theory of structural discrimination (ie, ways in which institutional policies and/or practices may constrain people's rights and opportunities) to investigate access to mental health treatment for disadvantaged groups. Besides institutional policies and legislations, Yang et al revealed that structural discrimination can also manifest through local cultural processes that interact with structural forces to create context-related dynamics of stigma and health inequities, ${ }^{41 p 85}$. Yang et al used Kleinman's concept of 'moral experience' of culture to investigate 'what matters most' to individuals who inhabit local social worlds. ${ }^{42}$ The concept of 'moral experience' or 'what matters most' for stakeholders in a local social world' provides a new theoretical model for explaining the social responses of stigmatisers and stigmatised alike. ${ }^{43}$ Yang and colleagues argue that culture influences stigma by threatening the ability of individuals to participate in the activities that govern 'what matters most' within a cultural context. The authors discovered that 'what mattered most' to ordinary individuals included, but not limited to, achieving status, health, money, relationships, livelihoods and religious experience. As stigma constrains the capability of individuals to participate in or uphold 'what matters most', stigmatised individuals (or their associates) are likely to lose their moral statuses and be excluded from local groups. This interdependence of moral status (and social capital) to stigma suggests ${ }^{44}$ the importance of their analysis for understanding the sources and consequences of leprosy-related stigma.

We therefore used the stigma models of Yang et al (2014), Corrigan et al (2005) and Link and Phelan (2001, 2004) to analyse the experiences of people with leprosy, to unravel how Yorùbá people stigmatised leprosy and people living with leprosy. Specifically, we used Yang et als concept of moral status to investigate how sociocultural connotations of leprosy impacted on the social status of those affected by leprosy, and the Yorùbá identity. Second, we used Corrigan $e t$ al s concept of structural discrimination to understand how disease control policies and religious practices interacted with cultural perceptions of leprosy to influence stigmatisation. Third, we used Link's and Phelan's concept of power differentials to explore whether the exclusion of people affected by leprosy was permanent and to identify contextual facilitators of inclusion among the Yorùbá people.

\section{RESULTS}

A total of 59 interviews were conducted with 47 participants (21 affected by leprosy and 26 community members). In all, 36 interviews $(61 \%)$ were conducted during phase 1 of fieldwork and the remaining 23 interviews $(39 \%)$ during phase 2 . The 21 participants affected by leprosy were aged 35 to 90 years; 11 were women. One of the 11 women and 8 of the 10 men were literate. Community member participants were aged 18 to 78 years; 6 (23\%) were women; all were literate. Tables 1 and 2 summarise demographic characteristics of participants affected by leprosy and community member participants, respectively. 


\begin{tabular}{ll}
$\begin{array}{l}\text { Table } 1 \\
\text { affected by leprosy }\end{array}$ \\
\hline Characteristics & $\begin{array}{l}\text { Value } \\
\text { (n=21) }\end{array}$ \\
\hline Age range (years) & $35-90$ \\
\hline Female & $11(52 \%)$ \\
\hline Literate (ie, at least primary education) & $9(43 \%)$ \\
Employed (trader, farmer, civil servant, etc) & $19(90 \%)$ \\
Visible physical impairment & $16(76 \%)$ \\
\hline Muslim & $7(33 \%)$ \\
\hline Christian & $14(67 \%)$ \\
\hline
\end{tabular}

See online supplementary appendixes 1 and 2 for additional details on demographic characteristics of participants.

\section{Connotations and significance of leprosy}

The Yorùbá word ètè usually translates as leprosy. Étè is used to describe the light-coloured or reddish patches on the surface of the skin and the distinguishable deformities of the hands and feet of people affected by leprosy. ${ }^{45} 46$ A synthesis of the diverse community perspectives identified three key connotations of leprosy among the Yorùbá people.

The leading connotation was the intense shame and disgrace associated with leprosy. The word for shame in Yorùbá is ẹté which appears to be same as the word for leprosy (ètè) except for the slight difference in orthographical symbols on the second syllable of the two words to reflect the variation in pronunciation of the terms. Related to shame was the discrediting image of leprosy in Western Nigeria. Community members commented that, 'leprosy destroys a person's name and reputation' (Étè dúró fún ibani l'órúko jẹ). Everyone interviewed for this research indicated that associating leprosy with immoral behaviour discredited not just the people affected by leprosy, it collectively tainted the Yorùbá people. Commenting on the moral significance of leprosy, a Muslim cleric said the following:

Leprosy is a dreadful disease that is comparable to being cloaked with shame (Àrùn tó dàbi wípé wợn ko àbùkù bo ènìyàn

Table 2 Demographic characteristics of community member participants

\begin{tabular}{ll}
\hline Characteristics & $\begin{array}{l}\text { Value } \\
(\mathbf{n = 2 6 )}\end{array}$ \\
\hline Age range (years) & $19-78$ \\
\hline Female & $6(23 \%)$ \\
\hline Literate & $26(100 \%)$ \\
\hline Employed & $18(69 \%)$ \\
\hline Visible physical impairment & $0(0 \%)$ \\
\hline Muslim & $4(15 \%)$ \\
\hline Christian & $22(85 \%)$ \\
\hline
\end{tabular}

$n i)$. It is regarded as a disease of shame...Anything that is associated with shame is detested by Yorùbá people. In fact, leprosy is the most detestable of detested conditions...The Yorùbá detest anything that tarnishes their reputation and leprosy tarnishes people's reputation. That is why leprosy is detested (Bàbá Túndé (Contributions by participants of this research are anonymized by masking the names of respondents, places and activities. The people interviewed are referred to by pseudonyms to protect their identities), Male, Cleric).

The above account suggests that the degree of stigmatisation of a condition is related to the extent that the condition discredits an individual's reputation or threatens cultural identity. By using a cloak of shame as a metaphor for leprosy, this interview extract implies that leprosy completely overshadows an individual's reputation and by extension threatens societal identity in a culture that idolises moral character. Labelling leprosy as most contemptible in Yorùbá culture also implies that it will attract the harshest stigmatisation. Internalising the above 'grid of meanings' about leprosy will undoubtedly provoke a feeling that those with leprosy bring shame on their families and communities.

Leprosy was also associated with filth, a connotation that seemingly originated from a Yorùbá religious belief that socialising with unhygienic persons tarnished the reputation of religious priests. Based on their religious training, Yorùbá priests were expected to avoid people with leprosy to safeguard their religious status:

The Yorùbá are scrupulous about self-hygiene. People who are meticulous about personal hygiene hate filth. The Yorùbá believe that leprosy is a filthy disease. From a religious point of view, it is believed filth tarnishes the spiritual status of a worshipper. So, the Yorùbá people have learned to avoid things that tarnish their reputation. This is the basis of the norm of avoidance of people affected by leprosy (Bàbá Onílè, Male Traditional Ruler).

The above quote illustrates how religious traditions can shape contemporary beliefs that socialising with affected people produces courtesy stigma, thus triggering a general avoidance of people with leprosy.

\section{Causation and transmission of leprosy}

We identified two broad ideas of causation of leprosy in Yorùbá culture: supernatural and natural causes. Most community members and persons affected by leprosy claimed leprosy was more likely to be caused via supernatural affliction than by natural means. Although the idea of supernatural causation was dominant in Western Nigeria, respondents also indicated that belief in natural causation was gaining popularity, especially following decades of public health campaigns and treatment of leprosy in Yorùbá towns. The demographic characteristics of respondents revealed a generational split in perception, with most people aged 18-39 years reporting that leprosy was contracted through natural causes while those aged 40-80 years believed leprosy was a supernatural condition deliberately inflicted on a person by sorcery. 
The range of natural causes of leprosy cited by respondents revolved around four themes. The majority of community members believed leprosy was hereditary, passed down from parents to their children in families that were susceptible to the disease. Another popular view was that leprosy was caused by air-droplet infection, transmitted to people living with affected persons in poorly ventilated houses. Most of those who believe in air-droplet infection were aged 18-39 years. Less popular modes of natural causation included casual contact with skin lesions and ulcers; and the sharing of cups, plates and bedding with persons suffering from leprosy.

\section{Sources of stigma in Western Nigeria}

For this study, sources of stigma refer to the places, persons, social processes, institutional practices etc., from which the public learn about and/or imbibe negative stereotypes and discriminatory attitudes. Our sociological understanding of stigma suggests the ways in which people react to illness or disease are strongly linked to broader social and cultural processes. We therefore reviewed archival documents for clues of discriminatory institutional policies and social practices that potentially shaped community perceptions and social reactions to leprosy in Western Nigeria. Our analysis identified three dimensions of institutional responses to leprosy. First, examples of messages disseminated from 1930s onwards by missionaries through Christian churches that were intended to counter traditional beliefs about leprosy stated the following:

Leprosy is not caused by some juju or curse put upon a person...It should be regarded as a disease which can be passed from one person to another as smallpox, yet taking months or years to develop instead of days. ${ }^{29}$

Although affected persons were previously 'looked upon as unclean' [i.e. unhygienic], there was no need to 'fear them because they cannot give you the disease unless you intimately live with them or handle their open wounds'. ${ }^{29}$

Second, health promotion messages embedded in primary school books (first published in the 1940s and still used by children aged 9-10 years) included information on 'taboos' and 'behavioural prohibitions', such as not sharing food, kitchen utensils and beddings with people affected by leprosy ${ }^{4747-76}$. The continuing endorsement of these books and the persistence of leprosy-related taboos in the memories of teenagers interviewed for this study contribute to our understanding of the social practices in Western Nigeria. Third, we identified a twofold format poster-leaflet commissioned by the government in 1955 to raise awareness about the 'danger' posed by leprosy. ${ }^{48}$ The lower half of the poster-leaflet (figure 1) depicted that it was dangerous to have casual contact with or share beddings and dishes with people affected by leprosy. ${ }^{49}$
It is plausible that depicting leprosy as a 'dangerous' and contagious disease continues to influence discriminatory ideas and practices towards leprosy in Western Nigeria.

\section{Perspectives on symptoms and signs of leprosy}

While most people affected by leprosy were knowledgeable about the symptoms and signs of leprosy, they, however, did not associate the early symptoms and signs of their illness with the biomedical condition. Instead, they attributed the early symptoms and signs to other common skin diseases, for example, ringworm, eczema and nettle rash, perhaps because the general community only recognises leprosy when someone has deformities and/or inflamed reddish skin lesions.

\section{Help-seeking by affected persons}

Analysis of help-seeking behaviours of people affected by leprosy suggested a hierarchical pathway of help-seeking, with most affected persons starting with self-treatment, followed by consulting traditional healers and finally consulting churches and/or Western medical practitioners. Only 2 of 21 participants affected by leprosy consulted traditional healers and faith healing before they were referred to a leprosy hospital. The dominance of supernatural causation in this culture meant that the primary help-seeking pathway for most people was consulting traditional healers followed ultimately by referrals to a hospital. As only two people reported using faith healing alone or in combination with traditional medicine, faith healing was considered an alternative help-seeking pathway for this research. There was a tendency to alternate between traditional medicine and treatment at a general hospital; or between traditional medicine and faith healing before eventual referral for treatment in a leprosy hospital.

Many of those who visited traditional healers reported consulting up to five different traditional healers before leaving them for a leprosy hospital. While our sample was purposively selected so that quantitative measures may be heavily biassed, it is informative to note that participants' total delay arising from self-treatment, consulting traditional healers and/or faith healers ranged from 3 months to 25 years before referral to a leprosy hospital for treatment. Women respondents were more likely to delay diagnosis and treatment. The link between stigma, delayed help-seeking and gender are highlighted in the discussion section.

\section{Cultural understanding of treatment and cure}

In explaining the difference between treatment and cure, the following quote describes the normative expectations of 'sickness behaviour' in Yorùbá culture:

When a person is ill, they are expected to submit themselves for care and during this process of care they are given some medicament. It is the receipt of this medicament that we call treatment. Without receiving the medicament, they cannot progress to a cure. If they do not take 

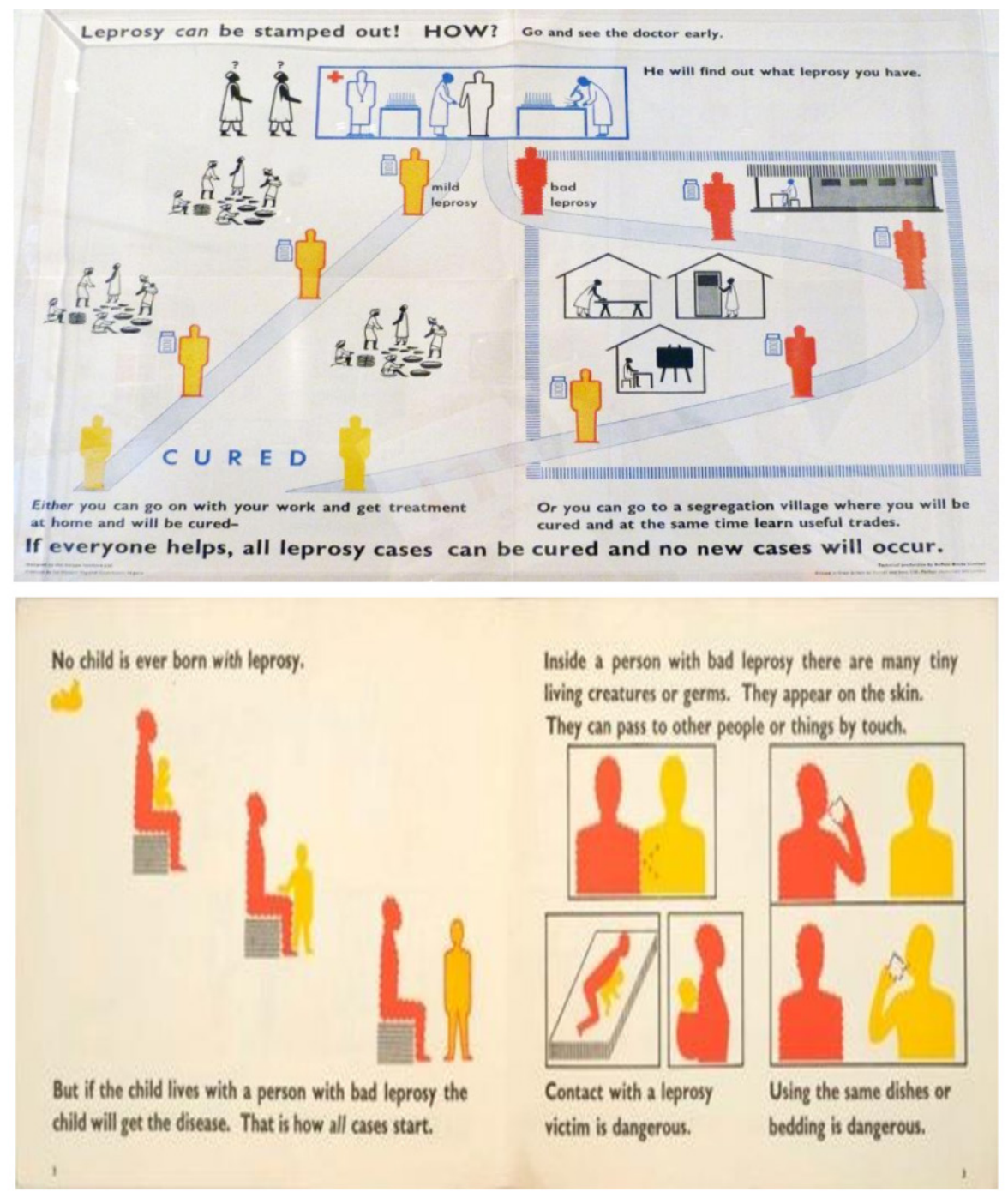

\section{Source: Otto and Marie Neurath Isotype Collection, University of Reading, (Reference number IC 7.1N)}

Figure 1 Poster-leaflet used in Western Nigeria in 1950s.

\begin{abstract}
the treatment, they will not be cured. Concerning leprosy, we can tell when someone is taking treatment, because we notice changes or differences in their body. For instance, if they had visible skin signs, these signs will begin to clear as they take their treatment. And we know that they are cured when their skin signs have completely disappeared, and they also feel well. So, cure is the complete removal of the signs of the disease from their body and blood. (Male, Farmer affected by leprosy)
\end{abstract}

This narrative captures the expected sequence of events starting from patients consulting or presenting themselves to a competent caregiver, to how they receive/are given medicaments to make the sick person better, through to completion of process of care indicated by the complete resolution of all visible signs of disease and perhaps, laboratory evidence of undetectable germs. Community member participants were emphatic that leprosy was treatable though a few people doubted that leprosy was completely curable. They implied the equivalence of 'being treatable' with the ability of Western medicine to kill leprosy bacilli and render affected persons noninfectious. An account supporting this viewpoint asserts the following:

Yes, leprosy is treatable to the extent that the disease is no longer in the body, but we know that the deformities caused by leprosy cannot be restored. The Yorùbá belief is that although leprosy is treatable, it is not completely curable. It is not completely curable because for instance, those who have deformities of the hands and feet cannot have their deformed fingers and toes restored to the normal state after medical treatment. Although it is possible to heal ulcers, we believe the fact that visible deformities cannot be restored means that leprosy is not completely curable (Bàbá Túndé, Male cleric).

Despite awareness of the efficacy of MDT to render affected persons noninfectious, Bàbá Túndé's account of cure refers to complete reversal of all signs of leprosy 
to their pre-disease state when neither skin lesions nor impairment are visible. This Yorùbá notion of cure is at variance with the biomedical understanding within global leprosy guidelines that interchanges the terms 'treatment' and 'cure' to indicate completion of a full course of MDT regardless of residual impairment ${ }^{50 \mathrm{p} 30}$. The biomedical understanding is based on laboratory evidence that MDT renders the patient noninfectious within a few days of starting of treatment, that is, reducing viable leprosy germs in the body to undetectable levels. ${ }^{51}$ The narratives in this section have implications for acceptance of those who have completed a course of antileprosy therapy in the community.

\section{The changing stigmatisation of people with leprosy in Western Nigeria}

A combination of community perceptions suggested that acceptance in Yorùbá denoted that 'a person affected by leprosy is unreservedly welcomed back into the community after completing antileprosy therapy; accorded their due dignity and respect; and granted the opportunity to participate in communal activities like other members of the community'. Respondents spoke about degrees of acceptance by alluding to 'full' as well as 'partial' acceptance of affected persons, emphasising that the symbolic meanings of leprosy (see section on connotations) prevented people affected by leprosy from being fully accepted.

Although popular perception in Yorùbá communities portrays affected persons as permanently stigmatised because of their perceived infectivity and immoral behaviour, interviews conducted for this research revealed that not all people affected by leprosy have had negative social experiences. A synthesis of the views of community member participants identified five factors that combined to stimulate acceptance of affected people in Western Nigeria: (i) treatment with antileprosy drugs; (ii) a good moral character; (iii) a supportive family network; (iv) financial contribution or a means of livelihood; and (v) contribution to community survival. These are briefly explained next.

\section{Role of antileprosy treatment in acceptance}

A dominant discourse was that treatment with antileprosy drugs was a prominent trigger of acceptance in Yorùbá communities, which is shown in the following quote:

\footnotetext{
It is possible to regain full acceptance when one has been treated for leprosy. Using myself as an example, I was shunned before I received treatment for leprosy. No one invited me to participate in family or communal activities because they thought I would infect them with leprosy. But after I was treated and discharged from MDT, they have welcomed me back into the family. I was also recalled by the community. Now we all participate together whenever there are ceremonies such as marriages (Female, Food vendor affected by leprosy).
}

This extract highlights the willingness of communities to accept those who have completed MDT, thus negating the view that leprosy was permanently stigmatised in Western Nigeria. Analysis of interview transcripts identified the emergence of a 'new narratives of acceptance' or possible life course available to persons with visible impairment and foot ulcers. The alternative accounts suggest that the people affected by leprosy in Western Nigeria have diverse life courses, allowing individuals to create new narratives to counteract existing cultural narratives that serve to marginalise them. The new narratives of acceptance build on four of five factors listed earlier: family support, financial contribution, a means of livelihood and a unique contribution to the survival of the community.

\section{Family support}

Our findings show that Yorùbá families had strong reciprocal bonds of kinship towards their members. Families upheld their obligations to relatives affected by leprosy despite the stereotypes of marginality in the general community by assisting affected persons to manage their lives in practical ways. Most individuals affected by leprosy described how they were supported by families from the moment of diagnosis through periods of treatment until after their discharge from treatment. Communal acceptance was however contingent on family acceptance:

Community acceptance depends on family acceptance. It persuades the community that the affected person has the backing of people fighting for her or his welfare...If the family performs its duty, the community will accept the affected person back despite their deformity. If the family welcomes the person, the community is more likely to re-accept them too (Male, Health worker affected by leprosy).

\section{Moral character}

While the previous quote underlined the role of family support in communal acceptance, community members also emphasised that only people with good moral characters were reaccepted by their families, implying that although families may be willing to accept affected persons, nonetheless, families can choose to neglect individuals with objectionable characters.

\section{Roles of financial contribution and livelihood}

Respondents acknowledged that for acceptance by the community, equally as important as family acceptance and moral character were money and a source of livelihood. A typical reference to the significance of financial contribution and livelihood in facilitating acceptance is as follows:

Those who have a means of survival are accepted back in the community. Once they can contribute financially to the community, they'll be accepted. You know 'money is the beacon of the heart's decisions' (Owó ni imórràn okàn): meaning money facilitates the resolution of complex problems. If they have money, they will be highly regarded by the community. If they have some change (i.e. money), they will have a voice in the family and community. Those 
who don't have money are not reckoned with...even if they don't have a stigmatizing disease (Female, Trader affected by leprosy).

The combination of money, a source of livelihood and financial contribution in the same account highlights that the importance of 'ability to contribute' to family and community needs to acceptance rather than the possession of money or a source of livelihood per se.

Our analysis has, thus far, focused on acceptance of affected individuals by families or the community. We now illustrate how groups of affected persons regained acceptance through their unique contribution to Yorùbá society.

\section{Contribution to community survival}

People affected by leprosy were barred from the markets in Yorùbá towns between the 1930s and 1960s due to the belief that they would infect the public with leprosy. This prohibition was repealed in the 1970s following public awareness of the curability and low infectivity of leprosy. Many respondents commented how affected persons earned the right to trade in Yorùbá markets due to the exceptional quality of farm products harvested from leprosy settlements and sold in the general markets. The unique advantage conferred by the superiority of their products thus triggered the collective acceptance of affected people in many Yorùbá towns. Asked about differences in community attitude towards people affected by leprosy today compared with 50 years earlier, one respondent claimed the following:

\begin{abstract}
To answer your question, yes, there is a positive change in public attitude to leprosy compared to say 40 to 50 years ago, when the public refused to trade with affected people or prevented them from selling in the market place. But today the ban on trading or mixing with affected people has been lifted and lorries now go into leprosy settlements to transport farm produce to the markets. People are keen to trade with people who have leprosy today because they recognise most affected persons who come to the markets have been treated and cured and do not pose a risk of transmitting the disease (Male, Traditional healer).
\end{abstract}

The above account reveals the increasing contemporary acceptance of people affected by leprosy and underlines the importance of public awareness for stimulating acceptance of affected people for their contribution to community development. The account also emphasises how practical considerations (contribution to food security in Yorùbá towns) facilitated the collective inclusion of those affected by leprosy into the community.

\section{DISCUSSION}

Even though leprosy has been associated with stigmatisation throughout history, there are variations in how people affected by leprosy are stigmatised around the world. Nancy Waxler ${ }^{52}$ and Zachary Gussow ${ }^{53}$ contend that stigma is closely linked to historical and sociocultural circumstances in which leprosy is found. Our study therefore adopted a qualitative, retrospective narrative life course approach to understand how leprosy shaped the lives of affected persons in Western Nigeria. We also drew on Yang et al's (2014) concept of moral status, ${ }^{414}$ Corrigan $e t a l$ s theory of structural discrimination ${ }^{40}$ and Link's and Phelan's concept of stigma ${ }^{34}$ to deepen social understandings of leprosy and responses to stigma. Following Yang et al, the initial paragraphs of our results section revealed how a moral association of leprosy and its deformity with intense shame and immoral behaviour was discrediting to the Yorùbá identity. The perception of leprosy as a symbolic problem that threatened communal survival generated public avoidance and exclusion of affected persons. This finding echoes aspects of Ronald Barrett's narrative study of leprosy in Northern India that showed how stigma led family members to expel relatives with deformities to isolated colonies and distant towns to beg for their survival. ${ }^{3}$ Barrett argued that the severity and intractability of leprosy stigma activated a cycle of concealment of the disease and treatment avoidance that, in turn, produced physical deformities and social discrimination that worsened the economic chances of people affected by leprosy. According to Barrett, the combination of visible deformities and begging for survival informed the cultural beliefs and stereotypes that have become the sources of stigma in India. On the contrary, other sources towhich researchers ${ }^{54}$ have attributed stigma include religious beliefs that consider leprosy to be a punishment forsins ${ }^{55}$, the treatment of leprosy inspecial hospitals ${ }^{53}$, and discriminatory laws.

Reverting to Western Nigeria, it is concerning that despite the seeming understanding of the biomedical causes and transmission of leprosy by sections of the Yorùbá community, people aged $>40$ years still believe in supernatural causation of leprosy, high infectivity and spread by casual contact with leprosy-affected people. While cultural beliefs and practices may perpetuate the views held by those $>40$ years of age, we contend that the discriminatory ideas endorsed by and disseminated through institutional policies and social practices (using religious teachings, primary school books and public health campaigns) also contributed to shaping contemporary views and responses to leprosy in Western Nigeria. Specifically, the equation of the mode of transmission of leprosy with that of smallpox, ${ }^{29}$ and the suggestion that leprosy is spread through casual contact with open wounds or sharing articles with affected persons ${ }^{27}$ may have transformed leprosy from an 'inherited disease' to a 'contagious disease'. These findings are similar to the introduction of stigma to northern Tanzania by Western public health educators in 1960s and 1970s, an intervention that instigated schoolchildren to avoid contact or sharing food with affected people. ${ }^{56}$ While leprosy was an unremarkable disease in northern Tanzania before the intervention, children later opposed the idea of affected persons living with relatives for fear that they would transmit leprosy to family members. 
We propose that although the Yorùbá people stigmatised leprosy prior to the arrival of Western missionaries, they nevertheless viewed leprosy as a disease transmitted among families who had a genetic disposition to leprosy, and not generally affecting others. In that sense, the Yorùbá were not afraid of catching leprosy from affected persons except when marriage with affected families was contemplated. However, after the introduction of medical ideas of infectivity, leprosy was transformed from an 'inherited disease' of a few families to a 'general threat' that was transmissible by casual contact. The idea of high infectivity thus amplified the dread of leprosy and prompted widespread avoidance of affected people because of the danger they now posed to society. It is easy to see how the circulation of 'authoritative' ideas of infectivity of leprosy and its link to smallpox may have influenced contemporary Yorùbá perception of causation and spread of leprosy. The continuing gap in understanding of the causes and transmission of leprosy presents a good opportunity for the leprosy control service to work with cultural, religious and educational institutions to de-emphasise the symbolic interpretation of leprosy in Western Nigeria and counteract the prevailing misconceptions. This should include reviewing and updating primary school curriculum, and Christian religious teachings identified as the underlying sources of stigma in Western Nigeria.

Three other issues remain in relation to recognising the symptoms of leprosy and seeking prompt treatment. First, the communal identification of leprosy by visible deformities prevents individuals and families from identifying early symptoms of the illness as leprosy. This has implications for help-seeking when such portrayal of leprosy is combined with belief in supernatural causation, leading to delays typically of many years between initial perception of symptoms and presentation to a leprosy hospital for diagnosis and treatment. Such delays can undoubtedly frustrate the achievement of the zero disability target of the WHO. Second, the Yorùbá conception of cure as 'complete resolution of all symptoms and signs of leprosy' perpetuates the idea of incurability of leprosy. Third, there are concerns about gender difference in help-seeking, as women were more likely to delay reporting for diagnosis and treatment. Other studies have highlighted that stigma disproportionately affects women in endemic countries with strong traditions, causing poor access to education and health services, and socioeconomic dependence, creating inferiority, marital rejection and isolation. ${ }^{57-60}$ We recommend a two-pronged approach to addressing long delays in Western Nigeria: (i) the leprosy control service should emphasise the message that 'even a single lesion can be a sign of leprosy and requires urgent attention to minimise the risk of impairment' rather than waiting for the person to develop several patches or visible deformities before referral is initiated and (ii) a gender-specific intervention to support and enable early reporting for diagnosis and treatment.
Contrary to the portrayal of leprosy as a social blemish that evokes permanent exclusion in Western Nigeria, this study revealed that stigmatisation of persons affected by leprosy is changing. There is enough evidence to show that a combination of factors (completion of MDT, good moral character, family support, livelihoods and contribution to food security) enables people affected by leprosy to resist marginalisation in Western Nigeria. These factors empower those affected by leprosy to meet social-transactional obligations in their community, thus raising their social status and facilitating their inclusion as fully viable members of society. ${ }^{61}$

Compared with overt exclusionary attitudes in northern India and in Western Nigeria, the context in Mali presents a more benign social response to leprosy. Drawing on archival sources and life history interviews with former patients in Mali, Eric Silla ${ }^{62}$ showed how contact with European institutions and other historical events influenced perceptions of leprosy and the identity of those affected by leprosy. Silla's ethnographic research revealed that although people affected by leprosy were shunned in precolonial Mali, villagers never expelled those who had skin patches. People with deformities or ulcers were given a separate hut in family compounds and in certain regions of Mali, they were prohibited from eating from the same bowl as others. Physical deformities excluded people from work and marriage (two key elements of social identity in agrarian societies), but leprosy never fully obscured all of one's other identities. Identities rooted in leprosy were remarkably transformed in colonial and postcolonial Mali. For instance, the policy of forceful segregation of patients in leprosaria and the separation of leprosy treatment from other healthcare services transformed all leprosy patients (whether they had deformities or not) into what Silla called 'social lepers'. In other words, medical treatment of leprosy in colonial Mali generated widespread social stigma, leading some patients to seek treatment in secret (Silla 1998, p156). To counter institutional discrimination, patient communities established around government leprosaria provided a foundation for collective social identities and the emergence of patient activists. Compared with the deprivation from social and economic participation experienced in rural Mali, patient activists used opportunities provided by their newfound communities to exert greater control over their lives.

Several historical events strengthened their destigmatisation efforts of people affected by leprosy and their quest to define their collective identity in Mali. These included the introduction of mass treatment with Dapsone by the WHO in 1951, followed by a call for the abolition of leprosaria and their replacement with mobile leprosy dispensaries (Silla 1998, p109). Equally important were Mali's attainment of political independence in 1960 and the growth of autonomous associations of Malians who were keen to participate in the new civil society. The passion for political activism subsequently led to the launch of a national association of persons affected by leprosy in 
1991, and to a national protest following a presidential candidate's insensitive rhetoric against people affected by leprosy. The association has facilitated the redress of injustices against people affected by leprosy including securing transparency over financial grants to people affected by leprosy and garden plots in the national capital, Bamako.

\section{CONCLUSION}

From the foregoing, we conclude that the goal of global zero stigma and discrimination will remain unachievable without better understanding of the cultural conceptualisations of leprosy, the sources and underlying drivers of stigma in each of the contexts where leprosy remains problematic. Ethnographic studies that explore the linkages between broader historical events and individual experiences of leprosy are essential for comprehending how stigma may have changed/is changing (or not) over time, and for developing and targeting context-specific interventions. Failure to identify the sources of stigma can encourage victim blaming of individuals and community members for stigmatising beliefs and practices. Our study identified the sources of stigma in Western Nigeria as societal-level factors such as institutional policies, legislations and practices that shaped community perceptions and social reactions to leprosy. Moreover, we identified a blend of stigma reduction interventions including antileprosy treatment, psychological and social support from families, and poverty reduction initiatives and involvement in community development initiatives. Finally, the historical events in Mali underline the importance of patient communities and of collective social action for changing social attitudes and redressing injustices against people affected by leprosy.

\section{Author affiliations \\ ${ }^{1}$ Nuffield Centre for International Health and Development, Leeds Institute of Health Sciences, University of Leeds, Leeds, UK \\ ${ }^{2}$ Nuffield Centre for International Health and Development, Leeds Institute of Health Sciences, University of Leeds, Leeds, UK \\ ${ }^{3}$ Social Sciences Building, University of Leeds, School of Sociology and Social Policy, Leeds, UK \\ ${ }^{4}$ Faculty of Social Sciences, Federal University Oye Ekiti, Department of Demography and Social Statistics, Ekiti, Nigeria \\ ${ }^{5}$ Faculty of Clinical Sciences, Lagos State University, Department of Behavioural Medicine, Ikeja, Nigeria}

Acknowledgements We thank the people affected by leprosy and the community members in Western Nigeria who participated in the study.

Contributors BE, JN and NE together conceived and designed the study. BE and $\mathrm{GA}$ conducted all interviews. All authors contributed to interpretation of data. $\mathrm{BE}$ led the writing of this paper with contributions from JN, NE, GA and BO. All authors read and approved the final version of the manuscript.

Funding This article presents independent research funded through the Overseas Research Students Awards (ORSAS) and International Research Scholarships from the University of Leeds, and a fieldwork grant from The Leprosy Mission. The funders had no involvement in the study design; collection, management, analysis and interpretation of data, writing of the report, and the decision to submit the report for publication.

Competing interests None declared.

Patient consent for publication Not required.
Ethics approval This study was approved by the University of Leeds Research Ethics Committee (Ethics Reference No: AREA 08-053) and the National Health Research Ethics Committee of the FMOH, Nigeria (Protocol Approval No: NHREC/01/01/200731/07/ 2009b). Informed consent was gained from all participants.

Provenance and peer review Not commissioned; externally peer reviewed.

Data sharing statement At the time of data collection, it was not the norm for qualitative evaluations to provide data sharing and as such participants did not give their consent to data sharing. We are, therefore, unable to provide access to raw data.

Open access This is an open access article distributed in accordance with the Creative Commons Attribution Non Commercial (CC BY-NC 4.0) license, which permits others to distribute, remix, adapt, build upon this work non-commercially, and license their derivative works on different terms, provided the original work is properly cited, appropriate credit is given, any changes made indicated, and the use is non-commercial. See: http://creativecommons.org/licenses/by-nc/4.0/.

\section{REFERENCES}

1. Nations MK, Lira GV, Catrib AMF, et al. Stigma, deforming metaphors and patients' moral experience of multibacillary leprosy in Sobral, Ceará state, Brazil. Cadernos de Saúde Pública 2009;25:1215-24.

2. Mull JD, Wood CS, Gans LP, et al. Culture and 'compliance' among leprosy patients in Pakistan. Soc Sci Med 1989;29:799-811.

3. Barrett R. Self-mortification and the stigma of leprosy in northern India. Med Anthropol Q 2005;19:216-30.

4. WHO. Leprosy update 2016: accelerating reduction of disease burden. world epidemiological record. . Geneva, Switzerland: WHO, 2016: 2017. 501-20.

5. WHO. Global leprosy strategy 2016-2020: accelerating towards a leprosy-free world - 2016 operational manual. New Delhi: WHO, 2016.

6. Rao PN. Global leprosy strategy 2016-2020: issues and concerns Indian Journal of Dermatology, Venereology and Leprology;2017:4-6.

7. Tiwari A, Suryawanshi P, Raikwar A, et al. Household expenditure on leprosy outpatient services in the Indian health system: a comparative study. PLoS Negl Trop Dis 2018;12:e0006181.

8. Sermrittirong S, Van Brakel WH. Stigma in leprosy: concepts, causes and determinants. Lepr Rev 2014;85:36-47.

9. Peters RMH, Dadun, Lusli M, et al. The meaning of leprosy and everyday experiences: an exploration in Cirebon, Indonesia. $J$ Trop Med 2013;2013:1-10.

10. Heijnders ML. Experiencing leprosy: perceiving and coping with leprosy and its treatment. A qualitative study conducted in Nepal. Lepr Rev 2004;75:327-37.

11. Peters RMH, Hofker ME, Van Brakel WH, et al. Narratives around concealment and agency for Stigma-reduction: a study of women affected by leprosy in Cirebon district, Indonesia. DCID 2014;25.

12. Rensen C, Bandyopadhyay S, Gopal PK, et al. Measuring leprosyrelated stigma - a pilot study to validate a toolkit of instruments. Disabil Rehabil 2011;33:711-9.

13. Peters RMH, Dadun, Van Brakel WH, et al. The cultural validation of two scales to assess social stigma in leprosy. PLoS Negl Trop Dis 2014;8:e3274.

14. Ibikunle PO, Nwokeji SC. Assessment of stigma among people living with Hansen's disease in south-east Nigeria. Lepr Rev 2017;88:43-57.

15. Ewhrudjakpor $\mathrm{C}$. Health care providers knowledge as correlates of their attitudes towards leprosy sufferers in Nigeria. Studies on EthnoMedicine 2008;2:115-20.

16. World Health Organization. mHealth: new horizons for health through mobile technologies: second global survey on eHealth. Switzerland: World Health Organization, 2011.

17. Awofeso N. Appraisal of the knowledge and attitude of Nigerian nurses toward leprosy. Lepr Rev 1992;63:169-72.

18. Iyor F. Knowledge and attitude of Nigeria Physitherapy students about leprosy. Asia Pacific Disability Rehabilitation Journal 2005;16:85-92.

19. Iyor F. Beliefs and attitudes about leprosy of non-leprosy patients in a reversely integrated Hospital. Asia Pacific Disability Rehabilitation Journal 2010;21:92-100.

20. Kleinman A, Hall-Clifford R. Stigma: a social, cultural and moral process. J Epidemiol Community Health 2009;63:418-9.

21. Leprosy N. Abuja: Nigeria centre for disease control, 2017. Available: http://www.ncdc.gov.ng/diseases/info/L [Accessed 20 Aug 2017].

22. Udo S, Chukwu J, Obasanya J. Leprosy situation in Nigeria. Lepr Rev 2013;84:229-37. 
23. Arulanantham S. Addressing inequality and exclusion - the opinion of people affected by leprosy in Africa and Asia, as to what should be included in any post Millennium Development goal framework. Leprosy Review 2014;85:1-9.

24. Abimbola K. Yoruba culture: a philosophical account. Birmingham, UK: Iroko Academic Publishers, 2006.

25. Ebenso B, Adeyemi G, Adegoke AO, et al. Using Indigenous proverbs to understand social knowledge and attitudes to leprosy among the Yoruba of Southwest Nigeria. J Afr Cult Stud 2012;24:208-22.

26. Mayer KU, Tuma NB. Event history analysis in life course research. Madison, Wisconsin: The University of Wisconsin Press, 1990.

27. Kummervold PE, Gammon D, Bergvik S, et al. Social support in a wired world: use of online mental health forums in Norway. Nord $J$ Psychiatry 2002;56:59-65.

28. Byrd J, Thompson L. "It's Safe to Ask": promoting patient safety through health literacy. Healthc Q 2008;11:91-4.

29. Williams I, Mears G, Raisor C, et al. An emergency medical services toolkit for improving systems of care for stroke in North Carolina. Prev Chronic Dis 2009;6.

30. Harden A, Thomas J. Methodological issues in combining diverse study types in systematic reviews. Int J Soc Res Methodol 2005;8:257-71.

31. Hammersley M. What's wrong with ethnography? London: Routledge, 1992.

32. Goffman E. Stigma: notes on the management of spoiled identity. London: Penguin, 1963.

33. Jacoby A. Epilepsy and stigma: an update and critical review. Curr Neurol Neurosci Rep 2008;8:339-44.

34. Link BG, Phelan JC, Stigma C. Conceptualizing stigma. Annu Rev Sociol 2001;27:363-85.

35. Scambler G. Re-framing stigma: felt and enacted stigma and challenges to the sociology of chronic and disabling conditions. Soc Theory Health 2004;2:29-46.

36. Link BG, Yang LH, Phelan JC, et al. Measuring mental illness stigma. Schizophr Bull 2004;30:511-41.

37. Jones EE, Farina A, Hastorf AH. Social stigma: the psychology of marked relationships. New York: W.H. Freeman, 1984.

38. Heatherton TF. The social psychology of stigma. Guilford Publications, 2003.

39. Pachankis JE, Hatzenbuehler ML, Wang K, et al. The burden of stigma on health and well-being: A taxonomy of concealment, course, Disruptiveness, Aesthetics, origin, and peril across 93 stigmas. Pers Soc Psychol Bull 2018;44:451-74.

40. Corrigan PW, Markowitz FE, Watson AC. Structural levels of mental illness stigma and discrimination. Schizophr Bull 2004;30:481-91.

41. Yang LH, Chen F-P, Sia KJ, et al. "What matters most:" a cultural mechanism moderating structural vulnerability and moral experience of mental illness stigma. Soc Sci Med 2014;103:84-93.

42. Kleinman A. What really matters: living a moral life amidst uncertainty and danger. Oxford: Oxford University Press, 2006.

43. Angermeyer MC, Carta MG, Matschinger H, et al. Cultural differences in stigma surrounding schizophrenia: comparison between central Europe and North Africa. Br J Psychiatry 2016;208:389-97.

44. Yang LH, Kleinman A. 'face' and the embodiment of stigma in China: the cases of schizophrenia and AIDS. Soc Sci Med 2008:67:398-408.

45. Church-Missionary-Society. A dictionary of the Yoruba language. 242. 2nd edn. Ibadan, Nigeria: University Press PLC, Ibadan, 2005.

46. Buckley A. Yoruba medicine. New York: Athelia Henrietta Press, 1997: 275.

47. Saxena S, Lora A, van Ommeren M, et al. Who's assessment instrument for mental health systems: collecting essential information for policy and service delivery. Psychiatr Serv 2007:58:816-21.

48. Heinrich R, Nemeth E, Pichler W, eds. Reaching the people: isotype beyond the west: Proceedings of the 33rd International Ludwig Wittgenstein-Symposium in Kirchberg. Image and imaging in philosophy, science and the arts. Lancaster: Ontos Verlag, 2010.

49. Neurath $\mathrm{O}$, Neurath $\mathrm{M}$. Isotype collection (reference number IC $7.1 \mathrm{~N}$ ) reading. University of Reading, 2012.

50. Raju MS, Rao PSS, Mutatkar RK. A study on community-based approaches to reduce leprosy stigma in India. Indian $\mathrm{J} L \mathrm{Lepr}$ 2008;80:267-73.

51. Kellagher M, Simpson J. Workload and workforce planning: developing a learning toolkit. Nurs Manag 1994;17.

52. Waxler N. Learning to be a leper: a case study in the social construction of illness. In: Mishler EG, ed. Social contexts of health, illness, and patient care. Cambridge: Cambridge University Press, 1981: 169-94.

53. Gussow Z. Leprosy, racism and public health: social policy in chronic disease control. San Francisco, CA: Westview Press, 1989.

54. Navon L, Beggars NL. Beggars, metaphors, and stigma: a missing link in the social history of leprosy. Soc Hist Med 1998;11:89-105.

55. Brody SN. The disease of the soul: leprosy in medievalliterature. Cornell University Press, 1974. ISBN: 0801408040, 9780801408045.

56. Hoffman Y, Everly GS, Werner D, et al. Identification and evaluation of mental health and psychosocial preparedness resources from the Centers for public health preparedness. Journal of Public Health Management and Practice 2005;11(Supplement):S138-S142.

57. Shale MJ. Women with leprosy. A woman with leprosy is in double jeopardy. Lepr Rev 2000;71:5-17.

58. Ulrich M, Zulueta AM, Cáceres-Dittmar G, et al. Leprosy in women: characteristics and repercussions. Soc Sci Med 1993;37:445-56.

59. Collins C, Green A. Public sector hospitals and organizational change: an agenda for policy analysis. Int J Health Plann Manage 1999;14:107-28.

60. Theobald S, MacPherson EE, Dean L, et al. 20 years of gender mainstreaming in health: lessons and reflections for the neglected tropical diseases community. BMJ Glob Health 2017;2.

61. Ebenso B, Ayuba M. 'Money is the vehicle of interaction': insight into social integration of people affected by leprosy in northern Nigeria. Lepr Rev 2010;81:99-110.

62. Silla E. People are not the same: leprosy and identity in twentiethcentury. Mali: Heinemann, 1998. 